\title{
Carriers Diffusion in GaAs/AlAs Type II Quantum Well
}

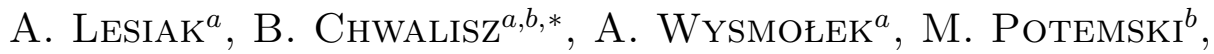 \\ R. StȩPNiEWski ${ }^{a}$ AND V. Thierry-Mieg ${ }^{c}$ \\ ${ }^{a}$ Institute of Experimental Physics, Warsaw University \\ Hoża 69, 00-681 Warsaw, Poland \\ ${ }^{b}$ Grenoble High Magnetic Field Laboratory, CNRS, Grenoble, France \\ ${ }^{c}$ Laboratoire de Photonique et de Nanostructures, CNRS, Marcoussis, France \\ The micro-photoluminescence of GaAs/AlAs type II double quantum \\ well structure is presented. The specific band alignment of the investigated \\ system allows obtaining high concentration of long lived carriers. This en- \\ ables us to study diffusion of carriers and/or indirect excitons. It was found \\ that the carrier flow does not follow the classical diffusion equation and is \\ driven by the potential modification due to the presence of photo created \\ carriers. \\ PACS numbers: 78.55.Cr, 78.66.Fd, 78.67.Hc, 75.75.+a
}

\section{Introduction}

GaAs/AlAs double quantum well (QW) structures are known to exhibit a number of intriguing properties [1]. This system allows achieving high carriers concentration, which makes it suitable for the observation of Bose-Einstein condensation of indirect excitons [2] or alternatively for the studies of super-fluid properties of dense electron-hole plasma [3]. In spite of the fact that the carriers diffusion was found to be important ingredient responsible for several phenomena observed in such structures [3], its detailed investigations are still desirable. In the present paper, we study the energy shift and the luminescence intensity of indirect transitions as a function of a distance from the excitation spot. The analysis of the obtained data allows for the discussion of possible mechanisms responsible for very effective flow of the photo-created electron-hole pairs.

*corresponding author; e-mail: barbara.chwalisz@fuw.edu.pl 


\section{Experimental details}

The investigated sample was grown by MBE technique. It is composed of GaAs/AlAs double QW, in which the $X$-symmetry electron state, localized in AlAs QW, has lower energy than that of $\Gamma$-symmetry state in GaAs QW. In order to compare the properties of indirect and direct recombination processes two additional GaAs/ $\mathrm{Ga}_{0.67} \mathrm{Al}_{0.33}$ As quantum wells were introduced into the structure. The potential profile of the structure is schematically shown in Fig. 1a. The micro-photoluminescence ( $\mu$-PL) imaging experiments were performed using a continuous flow cryostat in a temperature range of 4-100 K. A neodymium-YAG
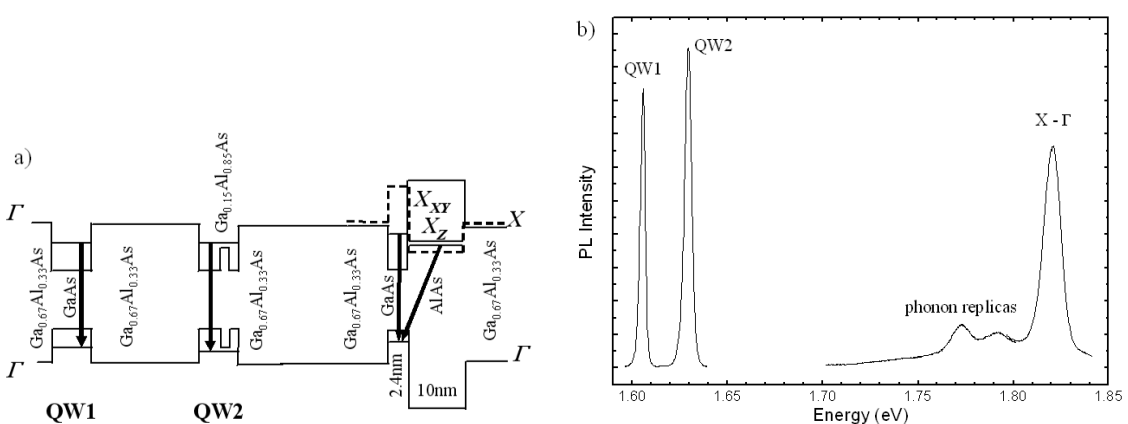

Fig. 1. (a) Scheme of the sample structure, (b) $\mu$-PL spectrum of the sample.

laser, operating at $514 \mathrm{~nm}$, was used for a continuous wave excitation. The maximum density of excitation power was approximately $700 \mathrm{~mW} / \mathrm{cm}^{2}$. The laser beam was focused on the sample using reflecting microscope objective with the magnification of 25. The resulted excitation spot size is estimated to be of about $5 \mu \mathrm{m}$ in diameter. PL emission was analyzed by a TRIAX 550 spectrometer equipped with CCD camera.

\section{Results and discussion}

The PL spectrum of the investigated sample is shown in Fig. 1b. It is dominated by the indirect $X-\Gamma$ emission in GaAs/AlAs double QW observed around $1.82 \mathrm{eV}$, followed by much less intense phonon replica. In the low energy part of the spectrum, two emission lines due to direct recombination in two additional quantum wells (QW1 and QW2) are observed, at $1.61 \mathrm{eV}$ and $1.62 \mathrm{eV}$, respectively.

In Fig. 2 the spectrally resolved $\mu$-PL image, representing spatial distribution of the energy and intensity of indirect $X-\Gamma$ transition.

It is clearly seen that the spatial dimensions of the region, from which indirect emission is observed, are much larger than those corresponding to the direct transitions in QW1 and QW2, which would serve as en estimation of the exciting laser spot diameter (see the inset in Fig. 2). This difference is caused by the fact 


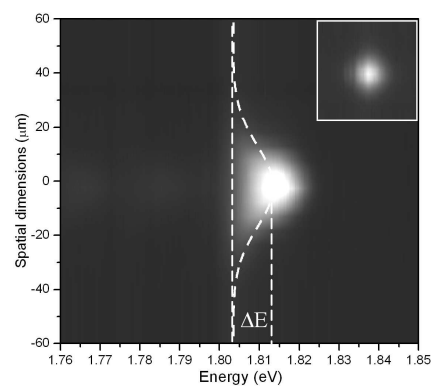

Fig. 2. The spectrally resolved $\mu$-PL images of the indirect transition. The vertical axis represents the spatial position on the sample in the quantum well plane, while the horizontal axis - the PL photon energy. $\Delta E$ corresponds to the emission energy shift. The inset shows corresponding dimensions of the exciting laser spot.

that for the indirect transition, due to its low recombination probability (resulting from spatial and $k$-space separation of electrons and holes), the carriers move apart from the excitation spot and recombine at a certain distance, up to $50 \mu \mathrm{m}$. Moreover, the observed emission energy gradually decreases as a function of the distance form the excitation spot.

The low temperature spectrally resolved $\mu$-PL images taken for different excitation powers are shown in Fig. 3. It is observed that with increasing excitation power the effective diffusion length rises up.
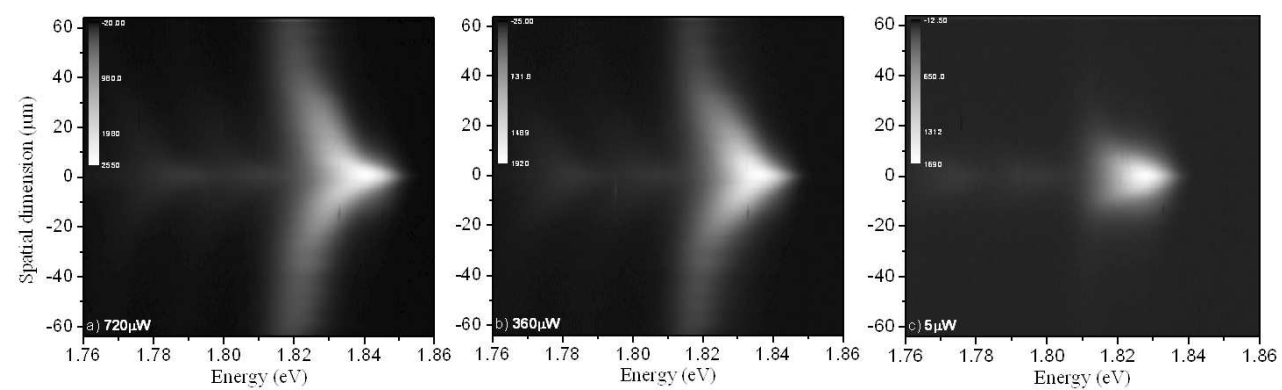

Fig. 3. The spectrally resolved $\mu$-PL images taken for indirect emission with different excitation power of (a) $720 \mu \mathrm{W}$, (b) $360 \mu \mathrm{W}$, (c) $5 \mu \mathrm{W}$, respectively.

It is also observed that the amplitude of the emission energy shift along the carrier flow ( $\Delta E$ in Fig. 2) increases with the increased excitation power.

The observed effects can be explained taking into account potential modifications caused by the electric field resulting from the electron hole separation that occurs in the indirect double QW structure. In the first approximation this effect should be proportional to the photo-created carrier concentration $(n)$ :

$\Delta E \propto n$. 
This causes that the energy shift depends upon actual carrier density at the recombination point. Assuming that the electron and hole concentrations are equal $(n=p)$ and that the emission intensity $I$ is proportional to their product, we can conclude that

$$
\Delta E \propto \sqrt{I}
$$

Moreover, taking into account that the emission intensity is proportional to the excitation power we find the relation between the emission energy shift and the excitation power

$$
\Delta E \propto \sqrt{P} .
$$

The above equation fits very well to the experimental data which is shown in Fig. 4.

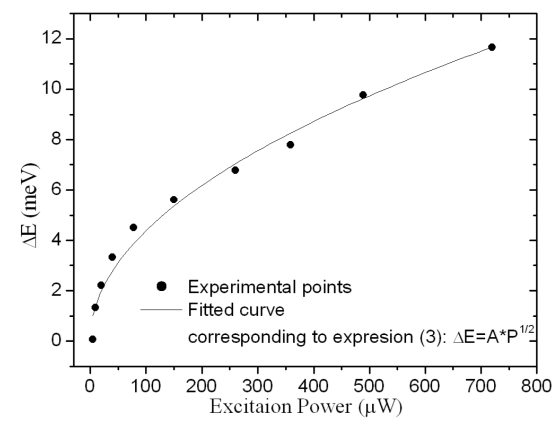

Fig. 4. The emission energy shift measured for different excitation powers. Solid line: fitted curve corresponding to Eq. (3).

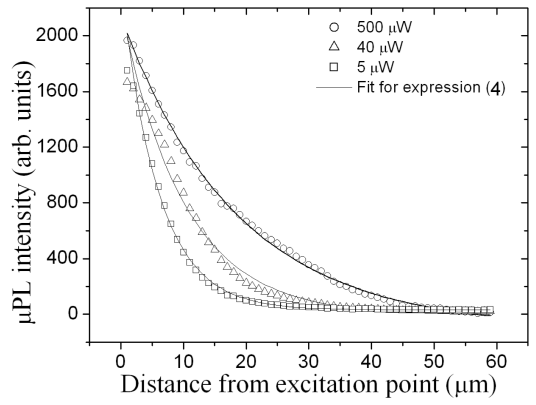

Fig. 5. Photoluminescence intensity measured as a function of the distance from the excitation spot. Symbols - experimental results measured with different excitation power, solid lines - exponential dependence according to Eq. (4).

In order to describe quantitatively the energy and intensity changes along the carrier flow we have fitted the luminescence intensity as a function of the distance 
from the excitation spot to the simple exponential dependence

$$
I(r)=A \exp (-r / \lambda),
$$

where $\lambda$-effective diffusion length, which is illustrated in Fig. 5. It was found that $\lambda$ is rising when increasing the excitation power (Fig. 6). These results suggest that classical diffusion does not explain the investigated effects. To describe the observed phenomena the additional contributions should be taken into account. As noticed above, the increased illumination influences the potential distribution in the excitation region (Eq. (3)). Thus, we can expect that due to the increased number of photo-created carriers the additional electric field will appear and will drive the carriers to spread out from the illuminated area. It can be expected the increased excitation power enhances the role of this mechanisms, which results in the longer effective diffusion length.

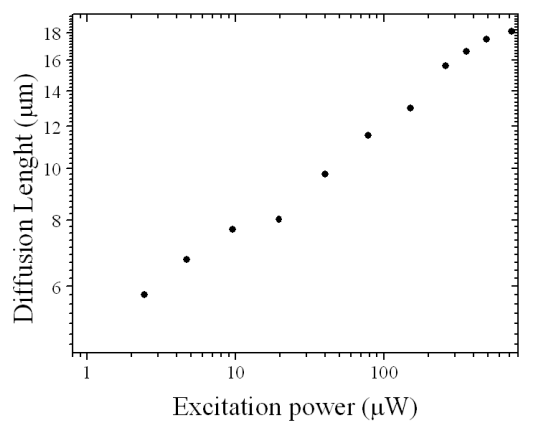

Fig. 6. The diffusion length as a function of the excitation power.
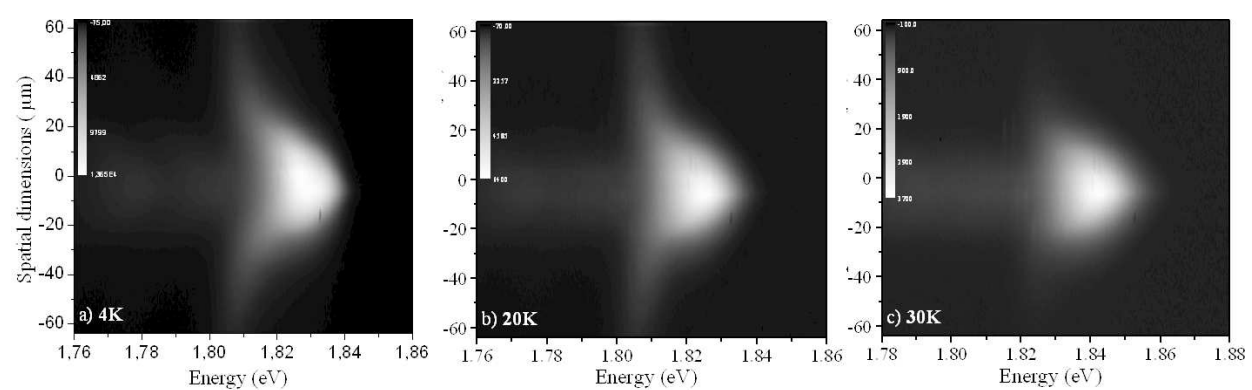

Fig. 7. The spectrally resolved $\mu$-PL images of the indirect transition measured for $4 \mathrm{~K}(\mathrm{a}), 20 \mathrm{~K}(\mathrm{~b}), 30 \mathrm{~K}$ (c), respectively.

The spectrally resolved $\mu$-PL images measured at different temperatures are shown in Fig. 7. It is observed that the effective length gets shorter upon rising temperature (Fig. 8) in contrary to the classical Einstein relation. This result could be understood in terms of an enhanced competition between indirect exciton 


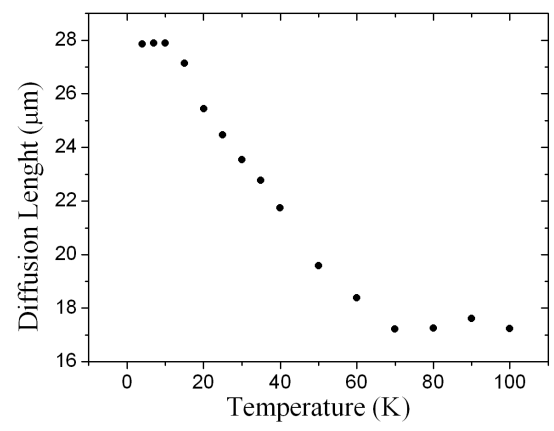

Fig. 8. The diffusion length as a function of the temperature.

recombination and other recombination processes. As a result the effective decay time of the indirect excitons decreases which leads to the shorter diffusion length.

\section{Conclusions}

The temperature and excitation dependence of the spatial distribution of the indirect emission provided important information about carrier flow in the investigated structure. The obtained results are hardly described by the classical diffusion equation. The observed carriers flow and the shift of indirect emission energy can be understood in terms of effective potential redistribution. The resulting potential shape, induced by illumination, drives carriers to flow in the quantum well plane. This observation is confirmed by experimental data obtained at elevated temperatures. In contrary to the prediction of the classical diffusion model the measured diffusion length decreases with increasing temperature.

\section{References}

[1] A. Wysmolek, M. Potemski, V. Thierry-Mieg, Physica E 12, 876 (2002); A. Wysmołek, B. Chwalisz, M. Potemski, R. Stȩpniewski, A. Babiński, S. Raymond, V. Thierry-Mieg, Acta Phys. Pol. A 106, 367 (2004).

[2] L.V. Butov, A. Zrenner, G. Abstreiter, G. Böhm, G. Weimann, Phys. Rev. Lett. 73, 304 (1994).

[3] D.W. Snoke, Y. Liu, Z. Vörös, L. Pfeiffer, K. West, Solid State Commun. 134, 37 (2005). 